\title{
Mechanisms of Dysregulated Antibody Response in Lyme Disease
}

\author{
Timothy J. Sellati ${ }^{1 *}$ and Dana M. Barberio ${ }^{2}$ \\ ${ }^{1}$ Global Lyme Alliance, Inc., Stamford, CT, United States, ${ }^{2}$ Edge Bioscience Communications, Sherborn, MA, United States
}

Keywords: Lyme disease, Borrelia burgdorferi, antigen-presenting cell, B cell, antibody

\section{INTRODUCTION}

OPEN ACCESS

Edited by:

John M. Leong,

Tufts University School of Medicine,

United States

Reviewed by:

Rachel Maurie Gerstein,

University of Massachusetts Medical

School, United States

Ronald Mark Wooten,

University of Toledo, United States

*Correspondence:

Timothy J. Sellati

timothy.sellati@globallymealliance.org

Specialty section:

This article was submitted to

Bacteria and Host,

a section of the journal

Frontiers in Cellular and Infection

Microbiology

Received: 29 May 2020

Accepted: 02 September 2020

Published: 07 October 2020

Citation:

Sellati TJ and Barberio DM (2020) Mechanisms of Dysregulated

Antibody Response in Lyme Disease.

Front. Cell. Infect. Microbiol.

10:567252

doi: $10.3389 /$ fcimb.2020.567252
Lyme disease (LD), caused by the spirochetal bacterium Borrelia burgdorferi, is transmitted by the black-legged tick Ixodes scapularis (Hu, 2016). LD is the fastest growing global tick-borne disease and annually affects $>300,000$ people in the U.S. alone (Steere et al., 2016). The economic impact is a staggering $\$ 1.3$ billion dollars per year (Adrion et al., 2015). LD can cause long-term, debilitating symptoms, including arthritis, carditis, and neurological complications (Hu, 2016; Steere et al., 2016). A longstanding question is why antibodies produced during primary infection are not able to completely clear spirochetes or confer protective immunity (Barbour et al., 2008). Antibody titers can remain for years in some LD patients while in others, they wane over time or never develop at all (Kalish et al., 2001). Herein, we describe animal studies that reveal mechanisms behind dysregulated development of adaptive immunity and provide insights that may be relevant to human immunity to B. burgdorferi infection.

\section{ROLE OF LYMPHOCYTES AND ANTIBODIES IN LYME DISEASE PATHOGENESIS}

Antibodies produced through $\mathrm{B}$ and $\mathrm{T}$ cell interactions either within or outside germinal centers are termed $\mathrm{T}$ cell-dependent (TD) whereas those produced without the aid of $\mathrm{T}$ cells are $\mathrm{T}$ cell-independent (TI). One mechanism whereby B. burgdorferi attempt to evade adaptive immunity is by continuously changing the sequence of a unique surface protein called variable major protein-like sequence (VlsE) (Norris, 2015). Such sequence variation generates a large repertoire of antigenically-distinct spirochetes that become unrecognizable to the antibodies that are mounted against a previous version of this protein. This may allow B. burgdorferi to persist for months or years if not effectively cleared through innate immune response and/or early diagnosis and treatment with antibiotics. Another defensive strategy relies on switching which immunogenic proteins are surface expressed [e.g., Outer Surface Protein A (OspA) and OspC], as spirochetes transit from one environment to another. OspA and OspC are predominately expressed when spirochetes are in the tick vs. the mammalian host, respectively. Notably, whole-proteome microarray analysis revealed that while relatively few $B$. burgdorferi open reading frames $(\sim 15 \%)$ encode immunogens, those that do elicit the same detectable antibodies in naturally infected humans and wild white-footed mice (Peromyscus leucopus), the predominant maintenance 
reservoir for B. burgdorferi (Barbour et al., 2008). Interestingly, a spectrum of disease severity has been observed in different mouse strains, reflecting their unique genetic composition, which controls the magnitude of humoral responses during $B$. burgdorferi infection (Weis et al., 1999).

Despite strong antibody responses in animals experimentallyinfected with $B$. burgdorferi and in many LD patients, this does not translate to robust disease-resolving and long-term immunity (Barthold and Bockenstedt, 1993; Aguero-Rosenfeld et al., 1996). In order to explore the mechanisms through which B. burgdorferi infection impacts the immune system and gain an understanding of the role of $\mathrm{B}$ and $\mathrm{T}$ cells in $\mathrm{LD}$ pathogenesis, researchers have conducted studies in mice lacking either or both of these lymphocyte populations.

Pathologies associated with B. burgdorferi infection of mice often spontaneously resolve, although animals may never completely clear spirochetes. In contrast, after infection with B. burgdorferi, severe combined immunodeficient (SCID) and recombination activating gene (RAG)-deficient mice, both of which lack $\mathrm{B}$ and $\mathrm{T}$ cells, developed severe, persistent arthritis that remained unresolved (Hastey et al., 2012). While $\mathrm{B}$ cells regulate disease progression and resolution in wildtype mice (McKisic and Barthold, 2000), adoptive transfer of $\mathrm{CD} 4+\mathrm{T}$ cells into RAG-deficient mice prior to B. burgdorferi infection increased arthritis and carditis severity (unless B cells were co-transferred), and CD8 $+\mathrm{T}$ cell transfer increased arthritis severity (McKisic et al., 2000). Conversely, adoptive transfer of serum from immunocompetent B. burgdorferiinfected mice into SCID mice ameliorated both arthritis and carditis (Barthold et al., 1997; McKisic and Barthold, 2000). Transfer of immune serum into naive recipient mice either prior to or at the time of inoculation also prevented $B$. burgdorferi infection (Barthold et al., 1997). Immunization of mice with late-stage LD patient sera that demonstrated strong antibody reactivity to several $B$. burgdorferi proteins, including OspA and $\mathrm{B}$, provided partial protection against $B$. burgdorferi challenge (Fikrig et al., 1994). These findings reveal that humoral immune responses generated in experimentallyinfected mice and $\mathrm{LD}$ patients play an important role in the resolution of some of the most commonly reported clinical manifestations (arthritis and carditis), which are driven principally by activation of inflammatory $\mathrm{T}$ cells and release of potent inflammatory mediators.

Researchers found unusually strong and persistent TD and TI IgM antibody production in lymph nodes during early infection and in bone marrow later on in the course of murine infection (Hastey et al., 2012; Richards et al., 2015). IgG-secreting plasma cells, on the other hand, accumulate slowly in the bone marrow. Only about $50 \%$ of the IgG response is clearly TD and, coupled with $\operatorname{IgM}$, is thought to contribute to the reduction but not elimination of $B$. burgdorferi from tissues (Hodzic et al., 2003). This TD repertoire of IgG contributes minimally to longterm antibody-mediated immunity, unlike the typical humoral response to bacterial pathogens (Hastey et al., 2012; Tracy and Baumgarth, 2017).

\section{MECHANISM(S) OF IMMUNE DYSFUNCTION IN LYME DISEASE}

To dissect the mechanisms behind this dysregulated response, Hastey et al. (2012) elucidated distinct stages of altered immune response using a mouse model of $\mathrm{LD}$. In the first phase of infection, B cells accumulated in lymph nodes and induced antibodies in a TI manner and in the absence of germinal centers. In other infectious diseases, such as mumps and HIV, swollen lymph nodes are a frequent early symptom of infection. Normally, the areas in which $\mathrm{T}$ and $\mathrm{B}$ cells are found in lymph nodes are well-defined. However, in B. burgdorferiinfected mice, this typical architecture was disrupted, with loss of organized B cell follicles and T cell zones (Tunev et al., 2011). Deterioration of B cell follicles, between days 5 and 10 postinfection occurred together with the presence of spirochetes within the lymph nodes (Hastey et al., 2014). In addition, $\mathrm{B}$ cells began to accumulate in large numbers, reaching over $70 \%$ in some instances and disrupting normal $\mathrm{T} / \mathrm{B}$ cell ratios (Hastey et al., 2012).

In the second phase, roughly 2-3 weeks later, short-lived germinal centers developed in lymph nodes. These germinal centers gave rise to relatively few antibody-producing plasma cells within bone marrow, leading to a third phase in which plasma cells only slowly accumulated. Lymph node germinal centers disappeared about 1 month after infection, despite the continued presence of bacteria at these sites. Curiously, B cell accumulation occurred after, not before, destructive changes in lymph node morphology. This suggests that the Borrelia infection, not B cell accumulation, somehow drives lymphoid tissue atrophy (Hastey et al., 2014).

So, while $B$. burgdorferi infection prompts strong serum antibody levels, and titers increase over the course of infection, the antibody response is ultimately ineffective in completely eradicating spirochetes and/or establishing long-term immunity (Tunev et al., 2011; Hastey et al., 2012; Elsner et al., 2015). B. burgdorferi benefits from this maladaptive immune response. This premise is corroborated by a study of antibiotictreated human LD patients, which included patients who had persistent symptomatology and those who had returned to health within 6 months after diagnosis and treatment (Blum et al., 2018). The researchers focused on plasmablasts, activated B cells that mature into plasma cells within germinal centers. They found that patients who ultimately recovered their health, as compared to those with persistent symptoms, had significantly more plasmablasts during early infection. The researchers determined this by comparing the percentage of plasmablasts as a total of all $B$ cells at the initial clinic visit, during early infection (even before beginning Doxycycline therapy). In addition, patients who ultimately returned to health had significantly higher titers of antibodies to a diverse array of $B$. burgdorferi proteins (Blum et al., 2018). Taken together, this is evidence that B. burgdorferi infection redirects the adaptive immune system away from a long-term protective antibody response and toward a less 
efficacious, rapid and strong, though short-lived antibody response (Richards et al., 2015).

Interestingly, Hastey et al. (2014) also provided evidence that Borrelia infection itself may have broader immunosuppressive effects. They tested this by using influenza virus vaccination as a tool to study TD antibody responses. Two groups of mice were influenza-vaccinated, with one group being infected with $B$. burgdorferi while the other was not. For the first 3 weeks, both groups of mice produced similar amounts of influenza-specific IgG. However, by 4 weeks, and until the study ended at 26 weeks, the B. burgdorferi-infected animals made significantly less influenza-specific IgG than the uninfected mice. By 9 weeks post-infection, there were far fewer influenzaspecific antibody-secreting cells in the bone marrow of the Borrelia-infected animals compared to uninfected influenzavaccinated mice (Elsner et al., 2015). This finding engenders an intriguing question about whether LD might negatively impact vaccination efficacy.

\section{IMPLICATIONS FOR DIAGNOSTICS}

There is more to be done in exploring these mechanisms of dysregulated antibody response in LD patients and there are clear implications for development of improved diagnostic tests. Physicians often follow the CDC-recommended two-tiered testing algorithm to detect $B$. burgdorferi-specific antibodies in patients suspected of having LD (Marques, 2015). The firsttier test is an enzyme-linked immunosorbent assay or ELISA, and if results are positive or borderline, a confirmatory secondtier test is done; a Western immunoblot analysis to detect IgM and IgG antibodies that are specific for B. burgdorferi proteins. In theory, this test determines if a $B$. burgdorferi infection is active (Marques, 2015). However, this CDC-recommended serodiagnosis may be misleading. In a small study of 79 patients who no longer had symptoms, but had a history of LD with and without arthritis 10-20 years ago, researchers examined antibody titers using the CDC two-tiered test (Kalish et al., 2001). They found that 10 individuals (13\%) currently had IgM responses (reflecting initial exposure) to B. burgdorferi and 34 (43\%) had IgG reactivity (reflecting longer term exposure) to $B$. burgdorferi. Antibody titers were even higher for patients who had LD with arthritis (but were currently asymptomatic), as six of $39(15 \%)$ currently had IgM responses and 24 of $39(62 \%)$ had $\operatorname{IgG}$ reactivity. This trend also is seen in infected mice, where IgM antibody levels do not wane but stay relatively high along with the increased IgG response (Hastey et al., 2012).

Theoretically, it would be expected that all recovered patients would lack evidence of IgM and many or all would continue to have circulating IgG. The presence of IgM in $13 \%$ of patients would be cause for confusion for physicians as the presence of this class of antibody typically wanes with clearance of the pathogen and recovery from infection. Larger studies need to be done to confirm and to explain the reasons for the continued presence of IgM. Another consideration is that high antibody levels, as discussed, may only offer transient protection, with alterations in germinal center architecture and defective production of long-lived plasma cells and memory cells leading to poor immunoprotection in the long-term (Hastey et al., 2012; Elsner et al., 2015).

Future efforts in Lyme disease diagnosis need to focus on distinguishing between active and inactive infection and improving sensitivity in detecting early disease while maintaining high specificity. Diagnosis would be greatly enhanced with the development and broad adoption of point-of-care testing, and simplified diagnosis. Addition of antigen targets expressed very early in LD (e.g., VlsE1 and pepC10) to current antibodybased diagnostic testing procedures have enhanced performance of the diagnostic assays (Porwancher et al., 2011; Marques, 2015). Additionally, direct detection of B. burgdorferi antigens or nucleic acid rather than antibody testing may eventually be possible with the development of advanced technologies (Branda et al., 2018). Not only might direct detection of spirochetal components be indicative of active infection, but the presence of nucleic acids and certain antigens coincides with the earliest stage of infection, when B. burgdorferispecific antibodies have yet to be produced. Examples include B. burgdorferi DNA detected using PCR (Mosel et al., 2019) and antigens such as OspC (Ohnishi et al., 2001) or peptidoglycan (Jutras et al., 2019). OspC is expressed on spirochetes as they transit from tick to mammalian host (Ohnishi et al., 2001) while peptidoglycan has been shown to persist in patients long after antibiotic treatment has ceased and patients are theoretically cured of active infection (Jutras et al., 2019). The latter observation strongly supports the notion of persistence of $B$. burgdorferi after antibiotic treatment as peptidoglycan is only produced by metabolicallyactive spirochetes.

\section{DISCUSSION}

There has been significant progress in deciphering the mechanistic foundation of $B$. burgdorferi's impact on the adaptive immune response, specifically the $B$ cell response and antibody production. While B. burgdorferi initially elicits a strong immune response, the end result is a failure by the immune system to clear the infection. This could set the stage for B. burgdorferi's persistence (Tracy and Baumgarth, 2017), which may underlie chronic symptoms such as arthritis, carditis, and skin and neurological complications. The many animal studies conducted to date reveal that $B$. burgdorferi relies upon multiple strategies to evade and disrupt the normal functioning of the immune system. The end results are inhibition of effective $B$ cell responses, disruption of the formation of stable germinal centers, and dampening the production of optimally protective antibodies and establishment of long-term memory cell populations. Importantly, many of these same evasion strategies appear to be employed by $B$. burgdorferi in LD patients, particularly those suffering from persistent or chronic disease. These intriguing observations provide an excellent foundation and springboard for further animal and human studies, with the goal of increased 
understanding of LD pathogenesis, better diagnostics, and ultimately novel and more effective therapeutic options for long-suffering patients.

\section{AUTHOR CONTRIBUTIONS}

DB and TS contributed to the writing of this manuscript. All authors contributed to the article and approved the submitted version.

\section{REFERENCES}

Adrion, E. R., Aucott, J., Lemke, K. W., and Weiner, J. P. (2015). Health care costs, utilization and patterns of care following Lyme disease. PLOS ONE 10:e0116767. doi: 10.1371/journal.pone. 0116767

Aguero-Rosenfeld, M. E., Nowakowski, J., Bittker, S., Cooper, D., Nadelman, R. B., and Wormser, G. P. (1996). Evolution of the serologic response to Borrelia burgdorferi in treated patients with culture-confirmed erythema migrans. J. Clin. Microbiol. 34, 1-9.

Barbour, A. G., Jasinskas, A., Kayala, M. A., Davies, D. H., Steere, A. C., Baldi, P., et al. (2008). A genome-wide proteome array reveals a limited set of immunogens in natural infections of humans and white-footed mice with Borrelia burgdorferi. Infect. Immun. 76, 3374-3389. doi: 10.1128/IAI. 00048-08

Barthold, S. W., and Bockenstedt, L. K. (1993). Passive immunizing activity of sera from mice infected with Borrelia burgdorferi. Infect. Immun. 61, 4696-4702.

Barthold, S. W., Feng, S., Bockenstedt, L. K., Fikrig, E., and Feen, K. (1997). Protective and arthritis-resolving activity in sera of mice infected with Borrelia burgdorferi. Clin. Infect. Dis. 25, S9-S17.

Blum, L. K., Adamska, J. Z., Martin, D. S., Rebman, A. W., Elliott, S. E., Cao, R. R. L., et al. (2018). Robust B cell responses predict rapid resolution of Lyme disease. Front. Immunol. 9:1634. doi: 10.3389/fimmu.2018.01634

Branda, J. A., Body, B. A., Boyle, J., Branson, B. M., Dattwyler, R. J., Fikrig, E., et al. (2018). Advances in serodiagnostic testing for Lyme disease are at hand. Clin. Infect. Dis. 66, 1133-1139. doi: 10.1093/cid/cix943

Elsner, R. A., Hastey, C. J., Olsen, K. J., and Baumgarth, N. (2015). Suppression of long-lived humoral immunity following Borrelia burgdorferi infection. PLoS Pathog. 11:e1004976. doi: 10.1371/journal.ppat.1004976

Fikrig, E., Bockenstedt, L. K., Barthold, S. W., Chen, M., Tao, H., Ali-Salaam, P., et al. (1994). Sera from patients with chronic Lyme disease protect mice from Lyme borreliosis. J. Infect. Dis. 169, 568-574.

Hastey, C. J., Elsner, R. A., Barthold, S. W., and Baumgarth, N. (2012). Delays and diversions mark the development of B cell responses to Borrelia burgdorferi infection. J. Immunol. 188, 5612-5622. doi: 10.4049/jimmunol.1103735

Hastey, C. J., Ochoa, J., Olsen, K. J., Barthold, S. W., and Baumgarth, N. (2014). MyD88- and TRIF-independent induction of type I interferon drives naive $\mathrm{B}$ cell accumulation but not loss of lymph node architecture in Lyme disease. Infect. Immun. 82, 1548-1558. doi: 10.1128/IAI.00969-13

Hodzic, E., Feng, S., Freet, K. J., and Barthold, S. W. (2003). Borrelia burgdorferi population dynamics and prototype gene expression during infection of immunocompetent and immunodeficient mice. Infect. Immun. 71, 5042-5055. doi: 10.1128/IAI.71.9.5042-5055.2003

Hu, L. T. (2016). Lyme disease. Ann. Intern. Med. 165:677. doi: 10.7326/L16-0409

Jutras, B. L., Lochhead, R. B., Kloos, Z. A., Biboy, J., Strle, K., Booth, C. J., et al. (2019). Borrelia burgdorferi peptidoglycan is a persistent antigen in patients with Lyme arthritis. Proc. Natl. Acad. Sci. U.S.A. 116, 13498-13507. doi: 10.1073/pnas.190417011

Kalish, R. A., McHugh, G., Granquist, J., Shea, B., Ruthazer, R., Steere, A. C., et al. (2001). Persistence of immunoglobulin $M$ or immunoglobulin $\mathrm{G}$ antibody responses to Borrelia burgdorferi 10-20 years after active Lyme disease. Clin. Infect. Dis. 33, 780-785. doi: 10.1086/322669

Marques, A. R. (2015). Laboratory diagnosis of Lyme disease: advances and challenges. Infect. Dis. Clin. North Am. 29, 295-307. doi: 10.1016/j.idc.2015.02.005

\section{FUNDING}

Publication of this manuscript was financially supported by Global Lyme Alliance, Inc.

\section{ACKNOWLEDGMENTS}

We would like to acknowledge Drs. Mayla Hsu and Nicole Baumgarth for thoughtful review of this manuscript prior to its submission for review.

McKisic, M. D., and Barthold, S. W. (2000). T-cell-independent responses to Borrelia burgdorferi are critical for protective immunity and resolution of lyme disease. Infect. Immun. 68, 5190-5197.

McKisic, M. D., Redmond, W. L., and Barthold, S. W. (2000). Cutting edge: T cellmediated pathology in murine Lyme borreliosis. J. Immunol. 164, 6096-6099. doi: 10.4049/jimmunol.164.12.6096

Mosel, M. R., Carolan, H. E., Rebman, A. W., Castro, S., Massire, C., Ecker, D. J., et al. (2019). Molecular testing of serial blood specimens from patients with early Lyme disease during treatment reveals changing coinfection with mixtures of Borrelia burgdorferi genotypes. Antimicrob. Agents Chemother. 63:e00237-19. doi: 10.1128/AAC.00237-19

Norris S. (2015). "vls antigenic variation systems of lyme disease Borrelia: eluding host immunity through both random, segmental gene conversion and framework heterogeneity," in Mobile DNA III, eds N. Craig, M. Chandler, M. Gellert, A. Lambowitz, P. Rice, and S. Sandmeyer (Washington, DC: ASM Press), 471-489. doi: 10.1128/microbiolspec.MDNA3-0038-2014

Ohnishi, J., Piesman, J., and de Silva, A. M. (2001). Antigenic and genetic heterogeneity of Borrelia burgdorferi populations transmitted by ticks. Proc. Natl. Acad. Sci. U.S.A. 98, 670-675. doi: 10.1073/pnas.98.2.670

Porwancher, R. B., Hagerty, C. G., Fan, J., Landsberg, L., Johnson, B. J., Kopnitsky, M., et al. (2011). Multiplex immunoassay for Lyme disease using VlsE1-IgG and pepC10-IgM antibodies: improving test performance through bioinformatics. Clin. Vaccine Immunol. 18, 851-859. doi: 10.1128/CVI.00409-10

Richards, K. A., Chaves, F. A., and Sant, A. J. (2015). CD4+ T cells promote antibody production but not sustained affinity maturation during Borrelia burgdorferi infection. Infect. Immun. 83, 48-56. doi: 10.1128/IAI.02471-14

Steere, A. C., Strle, F., Wormser, G. P., Hu, L. T., Branda, J. A., Hovius, J. W. R., et al. (2016). Lyme borreliosis. Nat. Rev. Dis. Primers 2:16090. doi: $10.1038 /$ nrdp.2016.90

Tracy, K. E., and Baumgarth, N. (2017). Borrelia burgdorferi manipulates innate and adaptive immunity to establish persistence in rodent reservoir hosts. Front. Immunol. 8:116. doi: 10.3389/fimmu.2017.00116

Tunev, S. S., Hastey, C. J., Hodzic, E., Feng, S., Barthold, S. W., and Baumgarth, N. (2011). Lymphoadenopathy during lyme borreliosis is caused by spirochete migration-induced specific B cell activation. PLoS Pathog. 7:e1002066. doi: 10.1371/journal.ppat.1002066

Weis, J. J., McCracken, B. A., Ma, Y., Fairbairn, D., Roper, R. J., Morrison, T. B., et al. (1999). Identification of quantitative trait loci governing arthritis severity and humoral responses in the murine model of Lyme disease. J. Immunol. $162,948-956$.

Conflict of Interest: DB is the owner of Edge Bioscience Communications, a freelance, contract scientific/medical writing and consulting company.

The remaining author declares that the research was conducted in the absence of any commercial or financial relationships that could be construed as a potential conflict of interest.

Copyright ( $\odot 2020$ Sellati and Barberio. This is an open-access article distributed under the terms of the Creative Commons Attribution License (CC BY). The use, distribution or reproduction in other forums is permitted, provided the original author(s) and the copyright owner(s) are credited and that the original publication in this journal is cited, in accordance with accepted academic practice. No use, distribution or reproduction is permitted which does not comply with these terms. 